\title{
Discussion on the Characteristics and Heritage Protection of Bai People's Bawangbian in Dali
}

\author{
Hefang Liu, Wenlei Yang * \\ Baoshan University, Baoshan City 678000, Yunnan Province, China \\ ${ }^{*}$ Corresponding author: Wenlei Yang
}

Keywords: Bawangbian; Characteristics; Heritage Protection.

\begin{abstract}
Bawangbian is a kind of special sport item of Bai people. The inheritance and development of Bawangbian is an effective way for the continuation of it. As a traditional sport item, there must be restriction factors which block the development of Bawangbian. With Bawangbian as research subject, this paper mainly explore the influencing factors in the process of development, discuss and analyze the existing problems, so as to lay a theoretical foundation for the inheritance and development of Bawangbian.
\end{abstract}

\section{Introduction}

"Bawangbian Dance" is the most characteristic folk dance of Bai nationality, which everyone can participate in. The more the people, the warmer the atmosphere, and the more spectacular the scenes. During the performance, the Bawangbian will give out a rhythmic, crisp and sweet sound. There are hundreds of playing ways of Bawangbian, but it often accompanied by the male dance Octagonal Drum Dance, or Shuangfeiyan Dance, with the action coherent freely, resolute and vigorous, showing the beauty of the female and forming a unique style. As an important part of the cultural behavior of Bai People, Bawangbian Dance and other Bai's culture and art joint together to reflect the social life of Bai people. At the same time, Bawangbian Dance, which is not an isolate cultural phenomenon, is integrated in the social life of ethnic groups. The main point of this paper is to clarify and confirm the status of dance development in this period by combing the performance form of the Bai's "Bawangbian Dance". Based on the data collected during the field study, combined with a large amount of historical data, this paper analyzes the historical context of the development of "Bawangbian Dance", systematically summarizes its characteristics and influences, and explore its development process through the research idea of "history-culture-dance".

Bawangbian is a folk dance popular in our country, also known as Dalianxiang, Dahuagun, Money whip, etc. Bawangbian has a long history in our country, and it is widely spread in the ethnic Han, Bai, Yao, Buyi, Hani, Li, etc. The manufacture of Bawangbian is basically perforated in a short bamboo about 1 meter long, and embedded coins in both ends, and stick stickers on the bamboo instead of copper coins. Dancers hold the middle part of the whip, or hold a whip with each hand to beat the body's arms, legs, shoulder, waist, back or the ground. When there are two or more dancers, they often beat each other in rhyme, with strong technical skills. Dancers can tap out ebullient or unrestrained rhythm according to their own characteristics and emotions to express their feelings. Due to the differences in region and national culture, the playing methods of Bawangbian in different regions have their own styles and characteristics, such as Hubei’s "Xuehua Gaiding”and "Huanglong Chanyao", Shanxi's “Sandiantou” and Shaanxi’s "Chanyao Guida”, etc. There are different opinions of the origin of Bawangbian in Bai nationality. Some people said that the Bawangbian was evolved by the "mourning stick" in the funeral, and other people said that Bawangbian was developed from the "Lianxinag" which was introduced into Yunnan during the period of "bureaucratization of native officers" in Ming Dynasty. However, from the view that Bawangbian Dance has spread all over the world, as well as the similarities and historical evolution of dance movements, the second argument is more credible and convincing. But anyway, Bawangbian, as the folk dance preferred by the majority of the people in the area of Bai nationality, its development 
interact with various nationalities in our country. It is the product of social development and widespread in the Bai region, with its own unique charm. Different regions of Bawangbian have a distinctive regional color. For example, during the auspicious festival, building house, marriage, or large ethnic custom activities, Bawangbina Dance will be performed as an indispensable entertainment. Bawangbian has a long history in the Bai area of Dali. Besides, the Bawangbian in Dali, Jianchuan, and Eryuan has the most Bai characteristics. There are a large number of dance types and playing ways of Bawangbian in Bai nationality, among which "Yitiaojie", "Dasimen", "Jiaogoujiao", "Guotianqiao", "Sanchahua”, "Qingwabeng” and "Beikaobei”, etc. are the representative dance movements of Bawangbian in Bai nationality. Furthermore, There is no restriction on the number of people when dance.

Bawangbian in Dali area with the "Raosanlin" and "Longshideng" around Erhai Lake as the representatives, the Bawangbian Dance in this area appears more passionate, relaxed and unrestrained compared with Bawangbian Dance in other areas. The movement transition here is relatively easier, flexible and smooth. The two movements "twist" and "tremor" are highlighted particularly in the whole dance moves, with a kind of natural and unrestrained taste. In the Jianchuan area, Bawangbian Dance is represented by the Shaxi and Jinhua's "Longshideng" and "Shilong Bawangbian”, with a particularly strong rhythmic sense, along with steady and powerful movement. In Eryuan area, the Bawangbian dance is mainly represented by the "Tianjiale", "Chunwang Zhengyue" and "Longshideng". The dance style here is between the Dali and Jianchuan, with relatively gentle movements. The rhythm basically maintains the medium speed without significant change. Movements like "twist, tremor and sinking” are all included. Throughout the Bai people' Bawangbian dance, "twist, tremor and sinking" are its basic movements, and the dance styles in different regions have different temperament. Dancers hold the middle part of the whip to beat arms, legs, shoulders, waist, back and other body parts, and also rub, touch, drag, rely on the body's joints, or beat, kick, rub, hold, buckle with their feet or hands. These actions are conducted alternatively. Most feet movement in the dance are horse stance, bow stance, squat and hop, combined with the whip beating the inner and outer side of the soles of the feet. No matter how complicated the dance moves, twist, tremor and sinking are its rhythm and slightly bent knees and slightly sitting hip as the basic action, accompanied by distinctive rhythm for its characteristics. With the change in the rhythm of the dance, a sense of determination and heavy feeling would be perceived by people. The acceleration of the rhythm cause a cheerful atmosphere in the form, but the dance action is still heavy. Twist, tremor and sinking have distinctive characteristics in the action of Bawangbian Dance. (1) Twist: There are two meanings of "twist" in the Bawangbian Dance: one is the twist with the waist as the axis, and another is the twist between the arm and shoulder with the shoulder as the power point. (2) Tremor: the flexible vibration formed by the straight up and down movement of knee joint according to the rhythm. (3)Sinking: the heavy feeling brought by coming down. The basic action in the Bawangbian Dance are slightly bent knees and slightly sitting hip, accompanied by the "tremor" throughout the dance, so that the whole dance action becomes more heavy.

All in all, the formation of a folk art style is closely related to the political, economic and cultural quality of the nation's long-term survival and development. The different temperament and character of the general rhythm "twist, tremor, sinking" of Bawangbian Dance in the Bai area of Dali, Jianchuan and Eryuan are the exchange results of regional difference and national culture, suggesting the existence of cultural diversity. Each nation has its own dance, as each nation has its own unique customs, which is the crystallization of its national culture for thousands of years of development. As a long term folk dance in Bai nationality of Yunnan province, the Bawangbian dance pregnant with rich aroma of soil and mountain atmosphere around Erhai Lake in Dali. Its style is the reflection of Bai ethnic character, temperament, physiological and psychological customs on the art.

Bawangbian Dance is still mainly the traditional folk performances and entertainment programs in the rural area on holidays. In order to carry forward this outstanding Bai ethnic cultural heritage, Bawangbian Dance have experienced careful arrangement and creation and have become one of the fixed performances of the traditional culture of the Bai people, which makes people to understand the Bai's excellent traditional culture of Dali, and to feel the atmosphere of traditional culture of Bai 
nationality. Since the opening of the public cultural sites for free in 2012, in order to enliven the cultural life of the masses, and popularize the traditional Bai ethnic dance, Dali's cultural departments have organized professional personnel to orchestrate and promote Bawangbian square dance, which have achieved gratifying achievements at present. We are delighted to see that the Bawangbian dance has gained popularity. Not only the aged but the young people have begun to jump the Bawangbian square dance.

With the integration and mutual impact of global culture, the national folk dance of the Bai nationality in Dali also develop and innovate with the continuous development of human society and history. The industrious and intelligent people in Dali have created many colorful cultural heritages in this beautiful and fertile land, and made indelible and positive contribution to the rich and profound culture of the Chinese nation. Bawangbian dance, as a representative of the intangible cultural heritage of the Bai nationality, how to protect and inherit it, how to face the impact of foreign culture, which need us to face the reality and make innovations to protect its historical culture, and meanwhile accept the needs of the masses in the new era, so that it has a new vitality.

\section{Acknowledgements}

Project name: Baoshan University Research Fund Project: A Study on the Inheritance and Development of the Bai People’s Bawangbian in Dali. Project number: $16 B Z 005$.

\section{References}

[1] Lin EC. The Glutinous Rice Sculpture Technology and Its Heritage Protection. Folk Custom in China. 2010(1):57-59.

[2] Li WQ, Cao YJ. On the Inheritance and Protection of Taomin Flower. Northern Music. 2016(11):213-213.

[3] Liu WF. Study on the Inheritance and Protection of Chinese Traditional Drama. Literature \& Art Studies. 2013(2):64.

[4] Zhou SJ. Analysis of the Original Artistic Charm and Inheritance and Protection Value of Baijian Dance. Art and Literature for the Masses. 2014(5):156-156

[5] Li H, Rong BD. Heritage Protection and Value of North Dong's Love Songs. Yihai. 2013(5):235-236.

[6] Fu ZP. Heritage Protection and Innovative Development of Huize Folk Songs. National Music. 2011(2):65-68.

[7] Chen LQ. A Survey of Heiyi Zhuang Minority's Traditional Handicrafts and Their Imparting. Journal of Guangxi Teachers Education University (Philosophy and Social Science Edition). 2010, 31(1):14-19

[8] Gonggar gamma. Discussion on the heritage protection and development of the unique art of Ali - Xuan Dance. Tibetan Art Studies. 2016(3).

[9] Long YC. On the Structural Characteristics and Heritage Protection of Nanping Playing and Singing. Sichuan Theatre. 2011(5):91-92.

[10] He ZC. On the Inheritance and Protection of Tongdong Folk Songs. Art and Literature for the Masses. 2015(3):52-52. 